\title{
MAGNETIC GEOMETRY AND THE CONFINEMENT OF ELECTRICALLY CONDUCTING PLASMAS
}

\author{
L. Faddeev*\# and Antti J. Niemi** ${ }^{*}$ \\ ${ }^{*}$ St.Petersburg Branch of Steklov Mathematical Institute \\ Russian Academy of Sciences, Fontanka 27, St.Petersburg, Russia ${ }^{\ddagger}$ \\ ** Department of Theoretical Physics, Uppsala University \\ P.O. Box 803, S-75108, Uppsala, Sweden ${ }^{\ddagger}$ \\ and \\ ${ }^{\sharp}$ Helsinki Institute of Physics \\ P.O. Box 9, FIN-00014 University of Helsinki, Finland
}

\begin{abstract}
We develop an effective field theory approach to inspect the electromagnetic interactions in an electrically neutral plasma, with an equal number of negative and positive charge carriers. We argue that the static equilibrium configurations within the plasma are topologically stable solitons, that describe knotted and linked fluxtubes of helical magnetic fields.
\end{abstract}

$\ddagger$ permanent address

* E-mail: FADDEEV@PDMI.RAS.RU and FADDEEV@PHCU.HELSINKI.FI

** E-mail: NIEMI@TEORFYS.UU.SE 
Plasma comprises over 99.9 per cent of known matter in the Universe. However, among the different states of matter its physical properties are the least understood. This is largely due to a highly complex and nonlinear behaviour, which makes theoretical investigations quite difficult. Particularly notorious are the instabilities that hamper plasma confinement in thermonuclear fusion energy experiments [1].

In the present Letter we consider the electromagnetic interactions within a charge neutral plasma, with an equal number of negative and positive charge carriers. We propose a first principles field theory model to describe the fluid dynamical properties of this plasma, and find results that challenge certain widely held views on plasma behaviour. In particular, we argue that stable self-confining plasma filaments can exist, and are described by topologically nontrivial knotted solitons.

In magnetohydrodynamics [1] the geometrical properties of an electrically neutral plasma are conventionally described using a single-fluid approximation. The individual charged particles contribution is described collectively by the hydrostatic pressure $p$, which according to standard kinetic theory relates to the kinetic energies of the individual particles $p \propto m v^{2}$. The equation of motion then follows from the properties of the pertinent energy-momentum tensor $T_{\mu \nu}$, the spatial part of its divergence coincides with the external dissipative force which leads to the Navier-Stokes equation

$$
\rho \frac{d \overrightarrow{\mathbf{U}}}{d t}=-\nabla p+(\nabla \times \overrightarrow{\mathbf{B}}) \times \overrightarrow{\mathbf{B}}+\eta \nabla^{2} \overrightarrow{\mathbf{U}}^{2}
$$

Here $\overrightarrow{\mathbf{U}}$ is the bulk (center of mass) velocity of the plasma, and $\eta$ is the coefficient of viscosity. The plasma evolves according to (1), dissipating its kinetic energy by the viscous force. This force is present whenever the plasma is in motion but ceases when the plasma reaches a magnetostatic equilibrium configuration. In that limit the NavierStokes equation reduces to a balance relation between the gradient of the hydrostatic pressure and the magnetic force,

$$
\nabla p=(\nabla \times \overrightarrow{\mathbf{B}}) \times \overrightarrow{\mathbf{B}}
$$

Ideally, one might expect that under proper conditions a plasma in isolation becomes self-confined due to the currents that flow entirely within the plasma itself. But this appears to be excluded by a simple virial theorem [1] which suggests that any static plasma configuration in isolation is dissipative. As a consequence of such apparently inborn instabilities, strong external currents are then commonly introduced to confine a plasma in laboratory experiments.

We now argue that there are important non-linear effects which are not accounted for by a structureless mean field variable such as the pressure $p$. These nonlinearities have their origin in the electromagnetic interactions between the charged particles within the plasma. They remain hidden when the energy-momentum tensor relates to the kinetic energies of the individual particles, but become visible once we recall the familiar but 
nontrivial relation between the kinetic momentum $m \overrightarrow{\mathbf{v}}$ and the canonical momentum $\overrightarrow{\mathbf{p}}$ of a charged point particle,

$$
m \overrightarrow{\mathbf{v}}=\overrightarrow{\mathbf{p}}-e \overrightarrow{\mathbf{A}}
$$

where $\overrightarrow{\mathbf{A}}$ is the electromagnetic vector potential. We propose that when these electromagnetic forces within the plasma are properly accounted for, the ensuing field theory model has the potential of supporting stable soliton-like configurations which describe helical, self-confined structures within the plasma medium.

Our starting point is a natural kinetic field theory model of a two-component plasma of electromagnetically interacting charged point particles such as electrons and deuterons. In natural units the classical action is

$$
\begin{gathered}
S=\int d t d^{3} x\left[i \psi_{e}{ }^{*}\left(\partial_{t}+i e A_{t}\right) \psi_{e}+i \psi_{i}{ }^{*}\left(\partial_{t}-i e A_{t}\right) \psi_{i}-\frac{1}{2 m}\left|\left(\partial_{k}+i e A_{k}\right) \psi_{e}\right|^{2}\right. \\
\left.-\frac{1}{2 M}\left|\left(\partial_{k}-i e A_{k}\right) \psi_{i}\right|^{2}-\frac{1}{4} F_{\mu \nu}^{2}\right]
\end{gathered}
$$

As usual $F_{\mu \nu}=\partial_{\mu} A_{\nu}-\partial_{\nu} A_{\mu}$. The $\psi_{e}$ and $\psi_{i}$ are two (complex) non-relativistic fields for electrons and ions with masses $m$ and $M$ and electric charges $\pm e$, respectively. Notice that we describe both charged fields by macroscopic (Hartree-Fock) wave functions. This is adequate in the classical Bolzmannian limit which is relevant in conventional plasma scenarios [1]. The action (2) determines our first principles description of a nonrelativistic plasma. Its magnetohydrodynamical properties are governed by the pertinent energy-momentum tensor $T_{\mu \nu}$, which can be constructed from (2) in a standard manner. When we include the contributions that account for the bulk motion of the plasma medium, this leads to an appropriate version of the Navier-Stokes equation (1D). Here we are interested in the ensuing static equilibrium configurations. These configurations are local minima of the internal energy $E$, which is determined by the temporal $T_{00}$ component of the energy-momentum tensor. For a stationary plasma fluid (2) we get from (2)

$$
\begin{aligned}
E=\int d^{3} x\left[\frac{1}{2 \mu}\{\right. & \left.\sin ^{2} \alpha\left|\left(\partial_{k}+i e A_{k}\right) \psi_{e}\right|^{2}+\cos ^{2} \alpha\left|\left(\partial_{k}-i e A_{k}\right) \psi_{i}\right|^{2}\right\} \\
+ & \left.\frac{1}{2} B_{i}^{2}+g\left(\psi_{e}{ }^{*} \psi_{e}-\psi_{i}{ }^{*} \psi_{i}\right)^{2}\right]
\end{aligned}
$$

Here $\mu=m \cdot \sin ^{2} \alpha=M \cdot \cos ^{2} \alpha$ is the reduced mass and $B_{i}=\frac{1}{2} \epsilon_{i j k} F_{j k}$ is the magnetic field. The quartic potential is the remnant of the Coulomb interaction with $g$ an effective coupling constant. It emerges when we first use Gauss' law to eliminate the electric field, and then recall that in any realistic plasma the Debye screening radius is small in comparison to any characteristic length scale of interest.

The free energy (3i) is subjected to the conditions that the plasma is electrically neutral with an equal (large) number $n_{e}$ of electrons and $n_{i}$ of ions, $n_{e}=n_{i}$ and the 
total number of charge carriers in the volume $V$ remains intact $n_{e}+n_{i}=N$. These conditions can be implemented by adding appropriate chemical potential terms to (3) in the usual fashion. But for simplicity we here account for them as constraints, imposed by appropriate boundary conditions. Besides the terms that we have displayed in (3) there can also be additional interaction terms for the charged fields. Such terms are usually induced by thermal fluctuations and finite density effects, or by gravitational interactions. However, according to standard universality arguments we expect the main features of (3) to persist at temperatures and distance scales which are relevant in conventional plasma scenarios.

We propose that (3) yields an adequate approximation for a non-relativistic plasma in a kinetic regime where the thermal energy is sufficiently high to prevent the formation of charge neutral bound states, which correspond to hydrogen atoms in the case of electrons and deuterons. Such bound states are present at lower temperatures, and their presence can be accounted for by terms of the form

$$
E_{b s}=\int d^{3} x\left[\frac{1}{2} \cdot \frac{1}{m+M}\left(\partial_{k} \Phi\right)^{2}+\lambda \cdot \Phi \psi_{e} \psi_{i}+\bar{\lambda} \cdot \Phi \psi_{e}^{*} \psi_{i}^{*}\right]
$$

Here $\Phi$ a real scalar field that describes a charge neutral bound state of $\psi_{e}$ and $\psi_{i}$. At a sufficiently high temperature this bound state degree of freedom decouples, and (3) becomes adequate for describing the bulk properties of the plasma.

Since $n_{e}=n_{i}$ we have overall charge neutrality. However, there can be local charge density fluctuations that should not be ignored. Indeed, we now proceed to argue that static charge density fluctuations are naturally present in (3). These fluctuations accompany stable, static solitons which describe filamental self-confined structures within the plasma. For this we first note that the different contributions in (3) respond differently to a scaling $\overrightarrow{\mathbf{x}} \rightarrow \lambda \overrightarrow{\mathbf{x}}$. The kinetic terms scale in proportion to $\lambda$ and the Coulomb potential in proportion to $\lambda^{3}$, but the magnetic energy scales like $\lambda^{-1}$. Consequently the existence of nontrivial, non-dissipative plasma configurations in (3) can not be excluded by simple virial arguments, quantitative investigations become necessary.

We start by observing that the vector potential $A_{k}$ enters at most quadratically. Consequently it can be eliminated: We vary (3) w.r.t. $A_{k}$ and get

$$
\begin{gathered}
A_{k}=\frac{1}{2 e} \cdot \frac{1}{\sin ^{2} \alpha\left|\psi_{e}\right|^{2}+\cos ^{2} \alpha\left|\psi_{i}\right|^{2}}\left[i \sin ^{2} \alpha \cdot\left(\psi_{e}{ }^{*} \partial_{k} \psi_{e}-\partial_{k} \psi_{e}{ }^{*} \psi_{e}\right)\right. \\
\left.-i \cos ^{2} \alpha \cdot\left(\psi_{i}{ }^{*} \partial_{k} \psi_{i}-\partial_{k} \psi_{i}{ }^{*} \psi_{i}\right)-\frac{2 \mu}{e} \cdot \epsilon_{k i j} \partial_{i} B_{j}\right]
\end{gathered}
$$

which determines $A_{k}$ in terms of an iterative gradient expansion, in powers of derivatives in the charged fields. We introduce new variables by

$$
\left(\psi_{e}, \psi_{i}\right)=\rho \cdot\left(\cos \alpha \cdot \sin \frac{\theta}{2} e^{i \varphi}, \sin \alpha \cdot \cos \frac{\theta}{2} e^{i \chi}\right)
$$


For reasons that will soon become obvious we have chosen these variables so that they are natural for describing tubular field configurations, with $\varphi$ and $\chi$ related to the toroidal and poloidal angles and $\theta$ a shape function that measures the distance away from the centerline of the tube. We compute the free energy (3) to the leading order in a selfconsistent gradient expansion, where we keep only terms which are at most fourth order in the derivatives of the variables (5). This approximation is adequate in conventional plasma scenarios where the fields are relatively slowly varying. We start by determining $A_{k}$ from (4) iteratively in the variables (5). We substitute the result in (3), and by defining a three-component unit vector $\overrightarrow{\mathbf{n}}=(\cos (\chi+\varphi) \sin \theta, \sin (\chi+\varphi) \sin \theta, \cos \theta)$ we finally get for the free energy

$E=\int d^{3} x\left[\frac{1}{2} \cdot \frac{1}{m+M} \cdot\left\{\left(\partial_{k} \rho\right)^{2}+\rho^{2} \cdot\left|\partial_{k} \overrightarrow{\mathbf{n}}\right|^{2}\right\}+\frac{1}{4 e^{2}}\left(\overrightarrow{\mathbf{n}} \cdot \partial_{i} \overrightarrow{\mathbf{n}} \times \partial_{j} \overrightarrow{\mathbf{n}}\right)^{2}+\frac{g \rho^{4}}{4}\left(n_{3}-\cos 2 \alpha\right)^{2}\right]$

We note that since $m$ and $M$ are both nonvanishing, overall charge neutrality implies that asymptotically $\theta \rightarrow 2 \alpha \neq n \pi$. Since $\rho \rightarrow$ const. $\neq 0$ asymptotically (see below), the Coulomb interaction then yields a mass term for the variable $\theta$. We also note that (6) naturally embodies a helical structure, described by the Hopf invariant [2]. To the relevant order in our gradient expansion

$$
Q_{H}=-\frac{1}{e^{2} 4 \pi^{2}} \int d^{3} x \overrightarrow{\mathbf{B}} \cdot \overrightarrow{\mathbf{A}}=-\int d^{3} x \nabla \cos \theta \cdot \frac{\nabla \varphi}{2 \pi} \times \frac{\nabla \chi}{2 \pi}=\Delta \varphi \cdot \Delta \chi
$$

Here $\Delta \varphi$ resp. $\Delta \chi$ denotes the $(2 \pi)$ change in the pertinent variable over the (would-be) tube, when we cover it once in the toroidal and poloidal directions over a magnetic flux surface with constant $\theta$.

The field $\rho$ is a measure of the particle density in the bulk of the plasma. If its average (asymptotic) value $\left\langle\rho^{2}\right\rangle=\rho_{0}^{2}$ becomes too small, the collective behaviour of the plasma will be lost and instead we have an individual-particle behaviour of the charged constituents, interacting via Coulomb collisions. Consequently we select the average $\rho_{0}^{2}$ so that it acquires a sufficiently large value in the medium. Local charge fluctuations then occur in regions where the unit vector $\overrightarrow{\mathbf{n}}$ becomes a variable so that $\theta \neq 2 \alpha$. According to our adiabatic approximation $\left|\partial_{k} \overrightarrow{\mathbf{n}}\right|$ is a slowly varying bounded function over the entire charge fluctuation region, and in particular it vanishes outside of the fluctuation region. When we inspect the $\rho$-equation of motion that follows from (6) we find that it can be related to a Schroedinger equation for the lowest energy scattering state in an external potential $\propto\left|\partial_{k} \overrightarrow{\mathbf{n}}\right|^{2}$. From this we then conclude that $|\rho(\overrightarrow{\mathbf{x}})|$ never vanishes; it is bounded from below by a non-vanishing positive value which is related to the ensuing scattering length. This implies that if we average the free energy (6) over $\rho(\overrightarrow{\mathbf{x}})$, to the relevant order in our gradient expansion the result can be related to the universality class determined by the Hamiltonian

$$
H=\int d^{3} x\left[\gamma \cdot\left|\partial_{k} \overrightarrow{\mathbf{n}}\right|^{2}+\frac{1}{4 e^{2}}\left(\overrightarrow{\mathbf{n}} \cdot \partial_{i} \overrightarrow{\mathbf{n}} \times \partial_{j} \overrightarrow{\mathbf{n}}\right)^{2}+\lambda \cdot\left(n_{3}-\cos 2 \alpha\right)^{2}\right]
$$


where $\gamma, \lambda$ are nonvanishing positive constants, proportional to the scattering length of our Schroedinger equation. This Hamiltonian is known to support stable knotlike solitons [2]. In particular, since the third (Coulomb) term is positive it does not interfere with the lower bound estimate derived in [4. This estimate states that the first two terms in (8) are bounded from below by the fractional power $\left|Q_{H}\right|^{3 / 4}$ of the Hopf invariant. Even though we do not expect that in the case of (6) this lower bound estimate remains valid as such, we nevertheless conclude that when $Q_{H} \neq 0$ the energy (6) admits a nontrivial lower bound; the conclusions from the virial theorem in [1] should not be adapted too hastily.

The properties of (8) with $\lambda=0$ have been studied in [2]-[8]. In particular, the numerical simulations in [7], 8] clearly confirm the existence of stable, knotted and linked solitons with a nontrivial Hopf invariant [2]. The present considerations firmly suggest that the conclusions in [2]-8] prevail also in the case of (6). Indeed, we have tentatively verified that similar solitons are present in (6), by numerically constructing a line vortex soliton in this model; we describe our solution in figure 1 . These solitons then become natural candidates for describing filamental and toroidal structures in the plasma, including coronal loops above the solar photosphere and the design of magnetic geometries in thermonuclear fusion energy experiments. The numerical simulations reported in [7]-9] are very extensive, and clearly reveal the complexity of the problem. Accordingly the interest has thus far mainly concentrated on the identification of soliton geometries, very little is still known about the solitons detailed physical properties. Consequently at this time we are not in a position to present definite physical predictions in the context of actual applications, high precision numerical methods still remain under active development [7], [8] and we have to limit ourselves to a few general remarks: In the numerical simulations that have been completed thus far, it has been found that for generic integer values $(\Delta \varphi, \Delta \chi)=(n, m)$ in (7) the $\lambda=0$ solitons of (8) form involved knotted and linked structures. Such complex geometries might be natural in a number of applications, for example when modelling coronal loops. But they might not be of any immediate practical interest for the design of plasma geometries in fusion energy experiments, where planar toroidal configurations are preferable. Indeed, there are also a few torus-shaped solitons which are essentially planar. These occur for values $(n, m)=(1,1),(2,1),(1,2),(2,2)[8$. The simplest one is $(1,1)$ but it appears to have an energy density that peaks at the toroidal symmetry axis. As such this may be an advantage in designing actual fusion reactors. But it could also become problematic, as it may interfere with the construction of an external torus-shaped coiling system which should be needed to create the soliton. On the other hand, the $(2,1)$ soliton seems to have a torus-shaped energy density distribution which vanishes at the symmetry axis and peaks at the centerline of the torus (see [8]). Since this soliton is also quite sturdy [8], it is a natural candidate e.g. for designing magnetic geometries for thermonuclear fusion energy purposes. In particular, this configuration strongly suggests that for a stable, toroidal planar geometry the safety factor [1] in the bulk of the plasma should not 
exceed $q \approx 2$. A configuration with a higher value for $q$ tends to adjust itself towards a geometrical shape which is not planar; see the computer animations in the www-address of reference [8].

In conclusion, we have argued that an electrically neutral conducting plasma can form stable, self-confining structures. This is due to soliton-like solutions, which we have shown will appear when we properly account for the nontrivial electromagnetic interactions within the plasma. We have proposed that our solitons can become relevant in a number of practical scenarios, including coronal loops and the design of magnetic geometries in thermonuclear fusion energy experiments. However, in order to assess the impact of our findings, detailed numerical investigations are necessary. Unfortunately the simulations remain highly complex, even with the present day supercomputers. Consequently we have not been able to reliably confirm that parameters such as the asymptotic density $\rho_{0}$ and the coupling $g$ can indeed be selected appropriately for the solitons to have direct technological relevance for example in the design of magnetic geometries for energy producing thermonuclear fusion reactors. But since over 99.9 per cent of all known matter in the Universe exists in the plasma state, there are no doubt numerous scenarios where our results can become important. Besides astrophysical applications or quark-gluon plasma experiments, these might include even an explanation to the highly elusive ball lightning.

We thank A. Alekseev, E. Babaev, A. Bondeson, H. Hansson, E. Langmann, V. Maslov, H.K. Moffatt, S. Nasir, A. Polychronakos, R. Ricca and G. Semenoff for discussions. We are particularly indebted to M. Lübcke for his help, and to J. Hietarinta for communicating the results in [8] prior to publication. We thank the Center for Scientific Computing in Espoo, Finland for the use of their computers. The work of L.F. has been supported by grants RFFR 99-01-00101 and INTAS 9606, and the work of A.J.N. has been supported by NFR Grant F-AA/FU 06821-308. 


\section{References}

[1] J. P. Freidberg, Ideal Magnetohydrodynamics Plenum Press, New York and London 1987; D. Biskamp, Nonlinear Magnetohydrodynamics Cambridge University Press, Cambridge 1993

[2] L. Faddeev, A.J. Niemi, Nature 387 (1997) 58; and Phys. Rev. Lett. 82 (1999) 1624

[3] L. Faddeev, Quantisation of Solitons, preprint IAS Print-75-QS70, 1975; and in Einstein and Several Contemporary Tendencies in the Field Theory of Elementary Particles in Relativity, Quanta and Cosmology vol. 1, M. Pantaleo, F. De Finis (eds.), Johnson Reprint, 1979

[4] A.F. Vakulenko, L.V. Kapitanski, Dokl. Akad. Nauk USSR 248810 (1979)

[5] A.J. Niemi Knots in interaction (Physical Review D, in press) hep-th/9902140

[6] J. Gladikowski, M. Hellmund, Phys. Rev. D56 (1997) 5194

[7] R. Battye, P. Sutcliffe, Phys. Rev. Lett. 81 (1998) 4798; and Proc. R. Soc. Lond. A455 (1999) 4305

[8] J. Hietarinta, P. Salo, Phys. Lett. B451 (1999) 60; and The ground state in the Faddeev-Skyrme model, University of Turku preprint, 1999; For video animations, see http://users.utu.fi/hietarin/knots/index.html

[9] M. Miettinen, A.J. Niemi, Yu. Stroganoff, Aspects of duality and confining strings (Physics Letters B, in press) hep-th/9908178 


\section{Figure Caption}

figure 1: An example of a numerically constructed tubular line vortex solution of (6), with energy density plotted as a function of the distance from the tubular centerline. We use standard cylindrical coordinates $(r, \phi, z)$ so that the tubular center-line coincides with the $z$-axis. For simplicity we have taken a limit of large ion mass which

sends $2 \alpha \rightarrow \pi$. All numerical parameters in (6) are $\mathcal{O}(1)$ and the helical structure is characterized by $\varphi+\chi=\phi+0.6 z$. 
figure 1

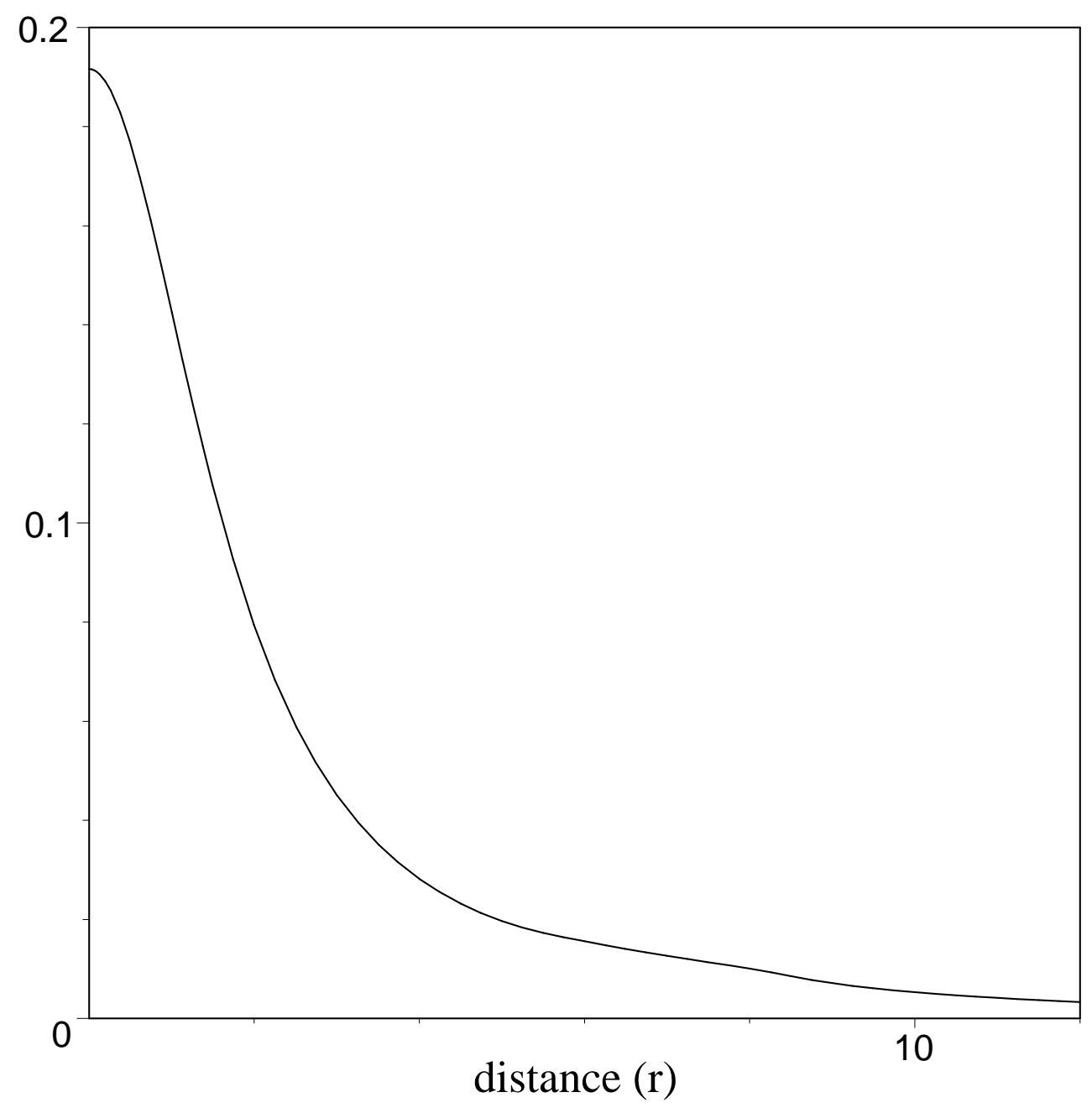

